\title{
Relevant logic and relation algebras
}

\author{
T. Kowalski \\ La Trobe University, Department of Mathematics and Statistics, \\ Melbourne, Victoria 3086, Australia \\ t.kowalski@latrobe.edu.au
}

\section{Introduction}

In 2007, Maddux observed (see [7]) that relevant logics have a natural interpretation in the language of relation algebras, with respect to which certain standard relevant logics are sound. He asked whether this interpretation was complete as well. In 2008, Mikulás proved in [10] that the relevant logic $\mathbf{R}$ was not complete with respect to square-increasing, commutative, representable relation algebras, answering thereby the original Maddux' question in the negative. Rather surprisingly, Maddux was able to show in [8] that another well-known relevant logic, namely RM, is complete with respect to idempotent, commutative, representable relation algebras. For several other results in similar vein, the reader is referred to Bimbó et al. [5] and Hirsch and Mikulás [4. The results are mostly negative, and they all focus on representable relation algebras.

In this note, I broaden the perspective a little and show that the answer to Maddux' question is in the positive, if one does not require representability. I was encouraged to do so by a surprisingly wide attention one little result of mine has received. This result (see [6]), motivated by Meyer's study [9] of the logic B, deals with weakly-associative relation algebras. Since these are obviously non-representable, relinquishing representability seemed natural.

\section{Relevant logic R}

We define the relevant logic $\mathbf{R}$ as a Hilbert system, (see [2, 1] and [12 for much more on relevant logics). As usual, formulas are built recursively from a countable set or propositional variables, by means of connectives $\wedge, \vee, \rightarrow$ and $\sim$. The axioms are:

1. $\alpha \rightarrow \alpha$

2. $(\alpha \rightarrow \beta) \rightarrow((\gamma \rightarrow \alpha) \rightarrow(\gamma \rightarrow \beta))$

3. $((\alpha \rightarrow(\alpha \rightarrow \beta) \rightarrow(\alpha \rightarrow \beta)$

4. $(\alpha \rightarrow(\beta \rightarrow \gamma)) \rightarrow(\beta \rightarrow(\alpha \rightarrow \gamma))$

5. $(\alpha \wedge \beta) \rightarrow \alpha,(\alpha \wedge \beta) \rightarrow \beta$

6. $((\alpha \rightarrow \beta) \wedge(\alpha \rightarrow \gamma)) \rightarrow(\alpha \rightarrow(\beta \wedge \gamma))$

7. $\alpha \rightarrow(\alpha \vee \beta), \beta \rightarrow(\alpha \vee \beta)$

8. $((\alpha \rightarrow \gamma) \wedge(\beta \rightarrow \gamma)) \rightarrow((\alpha \vee \beta) \rightarrow \gamma))$

9. $(\alpha \wedge(\beta \vee \gamma)) \rightarrow((\alpha \wedge \beta) \vee \gamma)$

10. $(\alpha \rightarrow \sim \alpha) \rightarrow \sim \alpha$ 
11. $(\alpha \rightarrow \sim \beta) \rightarrow(\beta \rightarrow \sim \alpha)$

12. $\sim \sim \alpha \rightarrow \alpha$

And the rules of inference are:

$$
\frac{\alpha \alpha \rightarrow \beta}{\beta} \quad \frac{\alpha \beta}{\alpha \wedge \beta}
$$

Then, $\mathbf{R}$ is defined as the smallest set of formulas containing all the axioms and closed under the rules of inference. A "multiplication" connective, called fusion, can be defined in $\mathbf{R}$ putting

$$
\alpha \cdot \beta \leftrightarrow \sim(\alpha \rightarrow \sim \beta)
$$

Since adding fusion to $\mathbf{R}$ is conservative, we assume that fusion is present from the outset, and count the defining formula above as one of the axioms of $\mathbf{R}$.

Sometimes $\mathbf{R}$ is further extended by adding an Ackermann constant, perhaps more informatively called truth constant, and requiring that it satisfies the axioms

- $t$

- $t \rightarrow(\alpha \rightarrow \alpha)$

Although extending $\mathbf{R}$ by the Ackermann constant is conservative as well, we will not assume the presence of $t$. In fact, we will assume the opposite, and on rare occasions where $t$ is present, we will use $\mathbf{R}_{t}$ to denote the extension.

\section{De Morgan monoids}

The usual algebraic semantics for $\mathbf{R}$ and $\mathbf{R}_{t}$ (see e.g., Dunn [3]) is provided by the class of De Morgan monoids. Out of a number of equivalent presentations of De Morgan monoids we choose the following. A De Morgan monoid is an algebra $\mathbf{A}=\langle A, \vee, \wedge, \cdot, \rightarrow, 1,0\rangle$ such that:

1. $\langle A, \vee, \wedge\rangle$ is a distributive lattice,

2. $\langle A, \cdot, 1\rangle$ is a commutative monoid,

3. the equivalence $a \cdot b \leq c$ iff $b \leq a \rightarrow c$ holds for all $a, b, c \in A$,

4. the following identities hold in A:
(a) $a \leq a \cdot a$
(b) $(a \rightarrow 0) \rightarrow 0=a$

In the terminology of residuated lattices (see [11]), a De Morgan monoid is an involutive, squareincreasing, distributive $\mathbf{F L}_{\mathbf{e}}$-algebra. As usual, we define $\sim x=x \rightarrow 0$ and $x+y=\sim(\sim x \cdot \sim y)$.

A De Morgan monoid $\mathbf{M}$ is normal if for every $a \in M$ exactly one of $a, \sim a$ belongs to $\uparrow 1$. If $\mathbf{M}$ is normal, then $\mathbf{M}$ is subdirectly irreducible. The converse does not hold, nevertheless normal De Morgan monoids generate the variety of De Morgan monoids.

For the embedding result, $\{\vee, \wedge, \cdot, \rightarrow, \sim\}$-reducts of De Morgan monoids will be important. We call them bare De Morgan monoids. 


\section{Relation algebras from normal De Morgan monoids}

Let $\mathbf{M}$ be a De Morgan monoid, and $X, Y \subseteq M$. We write $X \cdot Y$ or simply $X Y$ for the complex product of $X$ and $Y$, that is, $\{x y: x \in X, y \in Y\}$. Similarly, $X+Y=\{x+y: x \in X, y \in Y\}$ is the complex sum of $X$ and $Y$. Next, $\bar{X}$ is stands for $M \backslash X$. Finally, we let Fil(M) stand for the set of all proper filters of (the lattice reduct of) $\mathbf{M}$. Similarly, $\operatorname{Id}(\mathbf{M})$ will stand for the set of all proper ideals of $\mathbf{M}$. The following two lemmas are crucial for the construction to come.

Lemma 1. Let $\mathbf{M}$ be a subdirectly irreducible De Morgan monoid and $a, b \in M$. Let $F, G, H, P \in \operatorname{Fil}(\mathbf{M})$ be prime. The following hold:

1. If $F G H \subseteq P$ and $\bar{F}+\bar{G}+\bar{H} \subseteq \bar{P}$, then there exists a prime filter $R$ such that $G H \subseteq R$, $\bar{G}+\bar{H} \subseteq \bar{R}, F R \subseteq P$, and $\bar{F}+\bar{R} \subseteq \bar{P}$.

2. If $F G H \subseteq P$ and $\bar{F}+\bar{G}+\bar{H} \subseteq \bar{P}$, then there exists a prime filter $Q$ such that $F G \subseteq Q$, $\bar{F}+\bar{G} \subseteq \bar{Q}, Q H \subseteq P$, and $\bar{Q}+\bar{H} \subseteq \bar{P}$.

3. If $a b \in P$, then there exist prime filters $Q$ and $R$ such that $a \in Q, b \in R, Q R \subseteq P$, and $\bar{Q}+\bar{R} \subseteq \bar{P}$.

Lemma 2. Let $\mathbf{M}$ be a De Morgan monoid, $F$ a prime filter on $\mathbf{M}$, and I a prime ideal in $\mathbf{M}$. Then, the following hold:

1. $\sim F=\{\sim a \in M: a \in F\}$ is a prime ideal.

2. $\bar{F}=\{a \in F: a \notin F\}$ is a prime ideal.

3. $\sim I=\{\sim a \in M: a \in I\}$ is a prime filter.

4. $\bar{I}=\{a \in F: a \notin I\}$ is a prime filter.

5. $\overline{\sim F}=\sim \bar{F}$ is a prime filter.

6. $\overline{\sim I}=\sim \bar{I}$ is a prime ideal.

Now, let $\mathbf{M}$ be a normal De Morgan monoid, and $\mathcal{F}$ be the set of all prime filters of $\mathbf{M}$. Consider $U=\wp(\mathcal{F})$ as (a universe of) a Boolean algebra under the standard set-theoretical operations $\cup, \cap$ and - . Then $\operatorname{At}(U)=\{\{F\}: F \in \mathcal{F}\}$ is the set of atoms of $U$. Define a partial multiplication $\circ$ on $U$, putting

$$
\{F\} \circ\{G\}=\{P \in \mathcal{F}: F G \subseteq P, \bar{F}+\bar{G} \subseteq \bar{P}\} .
$$

and then extend it by

$$
u \circ w=\bigcup\{a \circ b: a, b \in \operatorname{At}(U), a \leq u, b \leq w\}
$$

onto the whole $U$. Next, define a unary operation ${ }^{`}$ on $U$ putting

$$
\{F\}^{\cup}=\{\overline{\sim F}\}
$$

for the atoms, and then setting

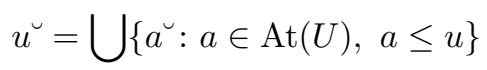

to extend it to the whole $U$.

Let $\mathbf{U}$ be the algebra $\left\langle U, \cup, \cap, \circ,\left\llcorner,-, 1^{\prime}, 0,1\right\rangle\right.$, where $\cup, \cap,-$ are the usual set-theoretical operations on $U=\wp(\mathcal{F})$, and $1^{\prime}=\{\uparrow 1\}, 0=\emptyset, 1=\mathcal{F}$. 
Theorem 1. The algebra $\mathbf{U}$ is a square-increasing, commutative, integral relation algebra.

For any normal subdirectly irreducible De Morgan monoid $\mathbf{M}$, we let $\mathbf{U}_{\mathbf{M}}$ stand for the relation algebra defined above. Consider a map $\varepsilon: M \rightarrow U_{\mathbf{M}}$ defined by

$$
\varepsilon(a)=\{F \in \mathcal{F}: a \in F\}
$$

Lemma 3. The map $\varepsilon$ embeds $\mathbf{M}$ as a bare De Morgan monoid into $\mathbf{U}_{\mathbf{M}}$.

Theorem 2. Every normal De Morgan monoid is embeddable as a bare De Morgan monoid into a square-increasing, commutative, integral relation algebra.

Corollary 1. The relevant logic $\mathbf{R}$ is sound and complete with respect to square-increasing, commutative, integral relation algebras.

This answers Maddux' question in the positive, without representability. It would be of considerable interest to obtain a characterisation of these De Morgan monoids for which the embedding construction produces a representable relation algebra.

\section{References}

[1] N. D. Belnap A. R. Anderson and J. M. Dunn. Entailment. The Logic of Relevance and Necessity, Vol. II. Princeton University Press, 1992.

[2] A. R. Anderson and N. D. Belnap. Entailment. The Logic of Relevance and Necessity, Vol. I. Princeton University Press, 1975.

[3] J. M. Dunn. The Algebra of Intensional Logics. PhD thesis, University of Pittsburgh, Penn. USA, 1966.

[4] R. Hirsch and S. Mikulás. Positive fragments of relevance logic and algebras of binary relations. Review of Symbolic Logic, 4:81, 2011.

[5] J. M. Dunn K. Bimbó and R. D. Maddux. Relevance logic and relation algebras. Review of Symbolic Logic, 2:102, 2009.

[6] T. Kowalski. Weakly associative relation algebras hold the key to the universe. Bulletin of the Section of Logic, 36:145, 2007.

[7] R. D. Maddux. Relevance logic and the calculus of relations. International Conference on Order, Algebra and Logics, Vanderbilt University, (abstract), June 2007. http://www.math.vanderbilt. edu/ oal2007/viewabstracts.php

[8] R. D. Maddux. Relevance logic and the calculus of relations. Review of Symbolic Logic, 3:41, 2010.

[9] Robert K. Meyer. Ternary relations and relevant semantics. Annals of Pure and Applied Logic, 127:195, 2004.

[10] S. Mikulás. Algebras of relations and relevance logic. Journal of Logic and Computation, 19:305, 2009.

[11] T. Kowalski N. Galatos, P. Jipsen and H. Ono. Residuated Lattices. An Algebraic Glimpse at Substructural Logics. Elsevier, 2007.

[12] R. Routley and R. K. Meyer. The semantics of entailment (i). In H. Leblanc, editor, Truth, Syntax and Modality, pages 199-243. North-Holland, 1973. 